\title{
Wireless MIMO Systems Employing Joint Turbo-Like STBC Codes With Bit-Level Algebraically-Interleaved URSCs
}

\author{
F. Mehran ${ }^{1}$, R. G. Maunder ${ }^{2}$ \\ ${ }^{1}$ School of EECE, University of Birmingham, Birmingham, West Midlands, UK \\ ${ }^{2}$ School of ECS, University of Southampton, Hampshire, UK \\ Email: farhad.mehran@ieee.org
}

\begin{abstract}
In this paper, permutations constructed based on algebraic derivations which are of particular interests due to better error-rate performance as well as simpler and practical hardware implementations, have been used in designing high performance fully-systematic joint space-time turbo coding technology. This scheme enjoys the integration of twin-/triplet-antenna bit-level space-time (ST) codes with the binary turbo-like codes of unpunctured codecs. The conducted performance evaluations reveal that this scheme has superior flare performance and yields additional coding gains in waterfall region, compared with the row-column block interleaved systems.
\end{abstract}

Index Terms - Channel coding, multiple-input multipleoutput (MIMO), space-time codes, turbo codes, wireless communications.

\section{INTRODUCTION}

Since the invention of multiple-antenna systems based on space-time block codes (STBCs) [1]-[3], they have evolved at an unprecedented rate and quickly employed in practical wireless applications and standards. In order to satisfy the reliability requirements in futuristic wireless communication systems wherein very low error-rates are required, they should be employed in conjunction with high performance channel codes for yielding more coding gains. The first class of channel codes with the near capacity performance has been introduced in [4]. They are parallel concatenated codes with iterative near maximum likelihood decoding (MLD) which are also referred to as turbo codes. Owning to their near capacity performance with feasible decoding complexities, they have been adopted in various wireless communication systems and standards including the UMTS/3GPP, CDMA2000/ 3GPP2, IEEE 802.16 (WiMAX), CCSDS, ETSI-DVB, and LTE systems. As a result of these dramatic evolutions in research and development of turbo codes and spacetime codes, the combined schemes have been studied [5],[6].

\section{SYSTEM MODEL}

Consider a point-to-point space-time turbo coded wireless communication system equipped with $N_{t}$ transmit and $N_{r}$ receive antennas (depicted in Fig. 1). Firstly, at the transmitter, the information bits are encoded by a turbo encoder which in a nutshell composed of digital IIR (infinite impulse response) filters (i.e. constituent codecs) and an interleaver $(\pi)$, and the coded signals are mapped to a particular signal constellation. Afterwards, the modulated signals are coded in space and time and transmitted over a wireless link. The signals at the receiver are noisy superposition of the transmitted signals corrupted by fading. The received signal vector can be expressed as $y=H . c+\gamma$ where $y=\left[y_{1}, \ldots, y_{N_{r}}\right]^{T}$ is an $N_{r}$ element vector of the received signals, $H$ is an $\left(N_{r} \times N_{t}\right)$ element channel matrix, $c=\left[c_{1}, \ldots, c_{N_{t}}\right]^{T}$ is an $N_{t}$-element vector of the transmitted signals, and $\gamma=\left[\gamma_{1}, \ldots, \gamma_{N_{r}}\right]^{T}$ is an $N_{r}$-element noise vector of the entries of Additive White Gaussian Noise (AWGN) process having a zero mean and a variance of an $N_{0} / 2$ per dimension. The received signals are combined and sent to maximum-likelihood detector, which are then inserted into the demodulator, and afterwards, the demodulated signals are fed into iterative near MLD decoder (composed of soft-input/-output component decoders, interleaver, and de-interleaver) for recovering transmitted information bits.

For the design of turbo coded systems, it has been reported from many contributions available on the literature that the increase in transmitted power does not lead to considerable reduction of bit-error-rate (BER) for the values on the orders of $10^{-5}$, which may be as high as $10^{-3}$ depends on the overall system design parameters. This BER flattening at medium to high signal-to-noise (SNR) ratios is shown to be a consequence of the relatively low free distance of the code. In futuristic wireless system generations, very low error rates will be required to open the way to demanding applications such as, e.g., multimedia services and Internet related contents. Hence, intensive research efforts have been dedicated by turbo coding community for finding strategies for combating error flooring effects i.e. find strategy for raising the minimum distance of the code. In [7],[8], Benedetto et al. introduced turbo-like codes based on serial concatenation which shown to perform better in flare region due to their higher minimum distances. Moreover, whether concatenated in serial or parallel, the minimum distance of the code which has critical role in systems' exceptional error-rate performance, is highly affected by the interleaver design. In particular, the performance of short-length turbo coded system is largely determined by the permutation type [9]. An interleaver is 
a single-input single-output casual device which generates the output sequence $\mathrm{C}=\cdots \mathrm{C}_{-1} \mathrm{C}_{0} \mathrm{C}_{1} \cdots=\cdots c_{\pi(-1)} c_{\pi(0)} c_{\pi(1)} \cdots$ i.e. a permutation of the input sequence $c=\cdots c_{-1} c_{0} c_{1} \cdots$. The permutation function $\pi$ denotes the time re-ordering on the input sequence indices i.e. the output symbol $C_{i}$ at the depth $i$ is the $\pi(i)$ th symbol $c_{\pi(i)}$ of the input sequence. Hence, for the interleaving entity of period $N_{I N T}: \pi(i)-$ $N_{I N T}=\pi\left(i-N_{I N T}\right), \forall i \in N^{N_{I N T}}=\left\{1, \ldots, N_{I N T}\right\} \quad$, the deinterleaver implements the reverse function that is $\pi^{-1}: N^{N_{I N T}} \rightarrow$ $N^{N_{I N T}}$ with $\pi^{-1}(\pi(\mathrm{i}))=\mathrm{i}, \forall i \in N^{N_{I N T}}[10]$. The structure of an interleaver in terms of its size and type plays a key role in determining the performance of turbo coded system in both waterfall and flare regions. Although using large interleaver sizes yield considerable coding gains, their long buffering delay is often too prohibitive to be practical in many applications.

An appealing alternative is to improve the minimum distance by devising more sophistical permutations which does not also incur any complexity penalty. However, designing such powerful permutations is not an easy task [11]. In particular, for turbo-like codes with serial structure which is the scheme of our interest due to improved flare performance, their type-specific interleavers have not enjoyed significant attention due to the reasons discussed in [12]. The most commonly used interleaver type in communication systems is block interleaver which has been extensively employed in turbo coded systems. The block interleaver (which is also termed rectangular or row-column interleaver) can be described by a matrix wherein the input bit sequences are permuted by filling a matrix row by row and shifting out column by column. To obtain a block interleaver function, the interleaver length should be first factorize as $N_{I N T}=$ $R \times C$ where $R$ and $C$ are termed depth and span of this array. Hence, the block permutation function is given by $\pi(i+j \times R+1)=i \times C+j+1, \forall i \in I=\{0,1, \ldots, R-1\}$ and $\forall j \in J=\{0,1, \ldots, C-1$, which implies that any two bits initially at a distance less than min $(I, J)$ will be situated at a distance superior to min $(I, J)$ after applying permutation function [10]. Although they shown to yield provisioning error rate performance especially when they have large and comparable depth and span [13], algebraically-derived aided design of permutation algorithm based on Costas arrays is a potential performance enhancement strategy due to the largest set of displacement vectors. Moreover, for the applications with large block lengths, using an algebraic permutation could be more efficient than the process of storing and reading out the sequences into/from the array with large dimensions. For the spreading factor of permutation function $\pi$ given by $\left\{\left(s_{1}, s_{2}\right)|| \pi(i)-\pi(\bar{i})\left|<s_{1} \rightarrow\right| i-\bar{i} \mid \geq s_{2}, \forall i \neq \bar{i}\right\}$ (i.e. if two symbols positioned within an interval of length $s_{1}$ in the input sequence are guaranteed to be separated by at least $s_{2}-1$ positions in the output sequence) one way is to

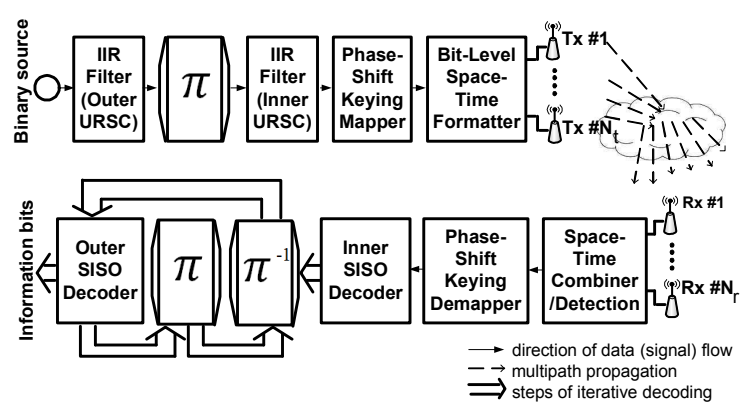

Fig. 1. Schematic of the point-to-point coded system with coherent phase shift keying when communicating over Rayleigh fading channels.

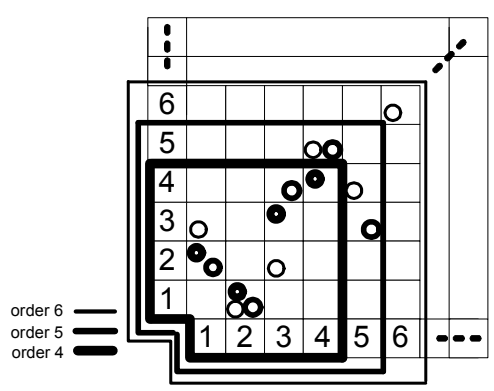

Fig. 2. The Costas arrays of orders 4,5 , and 6 .

express in terms of the displacement vector $(\Delta \alpha, \Delta \beta) \in Z^{2}$ of a pair of input values $i<\bar{i}$, a given permutation has spreading factor $\left(s_{1}, s_{2}\right)$ if wherever $\Delta \alpha<s_{1}$, then $|\Delta \beta| \geq$ $s_{2}$ [14]. To study the randomness of the permutor, the set of displacement vectors of a block interleaver can be used [14]: $D_{l}=\left\{(\Delta \alpha, \Delta \beta) \in Z^{2} \mid \Delta \alpha=\omega-x, \Delta \beta=\pi(\omega)-\pi(x)\right.$, $\left.0 \leq x<\omega<N_{I N T}+1\right\}$, for instance, the trivial identity permutation $\pi(x)=x$ has the smaller set of displacement vectors $D_{l}=\left\{(1,1),(2,2), \ldots,\left(N_{I N T}, N_{I N T}\right)\right\}$. Therefore, this set has $N_{I N T}$ elements. The largest set of displacement vectors could be attained for Costas arrays [15],[14] which are square arrays of dots/ $1 \mathrm{~s}$ and blanks/0s such that a) there exists exactly one dot/1 in each row and column; b) no four dots $1 \mathrm{~s}$ form a parallelogram; and c) no tree dots/1s lying on a straight line are equidistant [16], e.g. Fig. 2 depicts the Costas arrays of orders 4, 5, and 6 (note that there are also the other possible variations for each order). Let us begin with the definition of a Costas array: let $[n]:=\{1, \ldots, n\}, n \in N^{N_{I N T}}$ and consider a bijection $f:[n] \rightarrow[n] ; f$ is a Costas array iff $\forall i, j, k$ such that $1 \leq i, j, i+k, j+k \leq n: f(i+k)-f(i)=f(j+k)-f(j) \rightarrow$ $i=j$ or $k=0$ [17]. There exist two algebraic construction algorithms for the Costas permutations, known as the Welch construction and Golomb construction. Based on Welch construction [18],[16] and according to [19], let $p$ be a prime and $\vartheta$ be a primitive root of the finite field $F(p)$ of $p$ elements, and let $d \in[p-1]-1$ be a constant, then, the function $f:[p-1] \rightarrow[p-1]$ where $f(i)=\vartheta^{i-1+c} \bmod$ $p$ is a Costas permutation of order $p-1$. 


\section{ERROR PERFORMANCE EVALUATIONS: AlgORITHM AND DISCUSSIONS}

In this section, the performance of rate-one twinantenna space-time codes based on Alamouti's witty design [1], and half-rate triplet-antenna orthogonal STBCs based on [3], are presented in conjunction with the cascaded fully-systematic bit-interleaved recursive codecs employing aforementioned permutations. In Fig. 3, the flowchart diagram depicts a simplified version of the actual algorithm of the code used in the conducted simulations. For assessing the benefits of candidate physical layer (PHY) performance enhancement technologies to be employed in MIMO systems, an appropriate channel model should be used. In order to

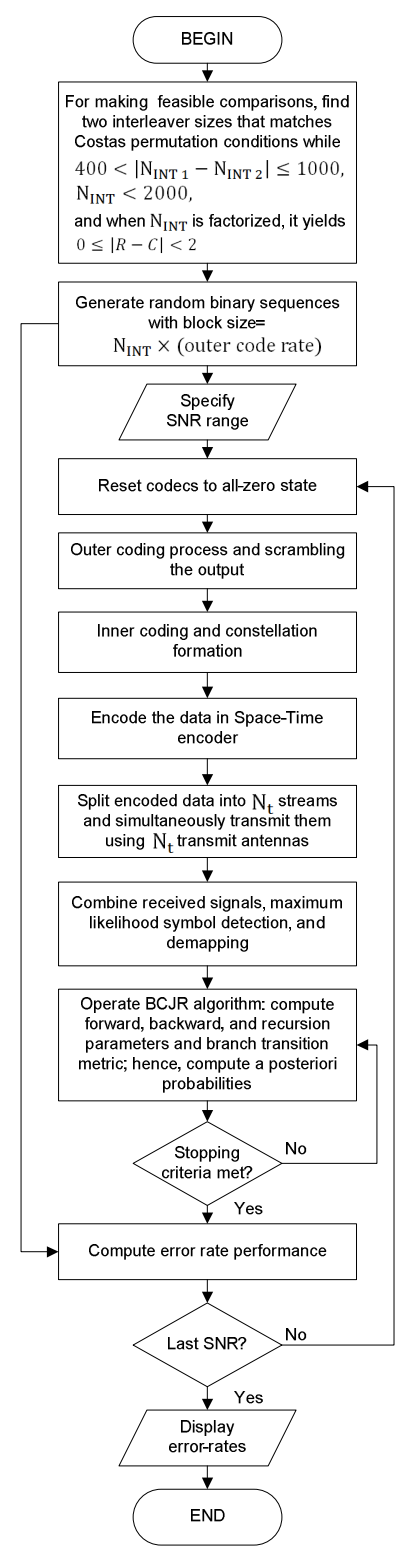

Fig. 3. A simplified flowchart of the code used in the simulations. assess the system performance, while for planning and deployment the location-specific models are used, the stochastic models are more commonly used for the design, testing, and type approval [20]. Hence, Rayleigh distribution which is the most commonly used model for describing the statistical time varying nature of the received signal's envelope in urban propagation environments with blocked line-of-sight, is utilized for predicting the probability density function of entries of channel matrix i.e. $h_{i j}(t)$ which is the channel impulse response between the $j^{\text {th }}$ transmit antenna and the $i^{\text {th }}$ receive antenna where $j \in\left\{1, \ldots, N_{t}\right\}$ and $i \in\left\{1, \ldots, N_{r}\right\}$. The signal constellations are formed by using coherent binary phase shift keying (BPSK) modulation. The constituent codecs are constructed based on the feedforward (FF) polynomials of $(111,101)_{2}$. Since in [21], it is suggested to use primitive feedback (FB) polynomial for raising the free distance of the code, in this design, we use FB polynomial $(111)_{2}$ in constituent codecs. The transmission sequences for space-time encoding for twin-antenna and triplet-antenna systems are given in (32) and (37) of [3]. At the reception side, the decision criteria expressed in (17)-(20) of [1] are used for detecting symbols of the system with two transmitting antennas. Meanwhile, the maximum likelihood detector amounts to minimize the decision metrics given in appendix of [2] for detecting symbols of the system with three transmitting antennas. For recovering the transmitted bits from the demodulated signal, the iterative decoder employs LogMAP algorithm. In Fig. 4 and Fig. 5, the associated bit error probabilities are shown versus the energy per bit/noise power spectral density $\left(E_{b} / N_{0}\right)$ for interleaver

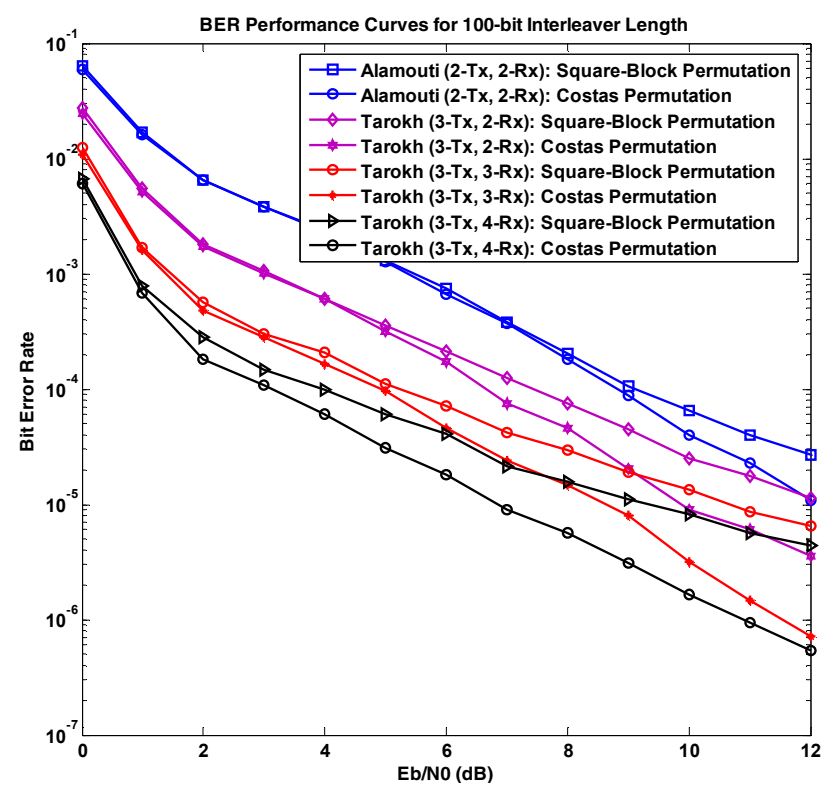

Fig. 4. Comparison of BER curves for MIMO systems with differing numbers of transmitter (Tx) and receiver ( $\mathrm{Rx})$ antennas, with Costas and Block permutations of $N_{I N T}=100$. 


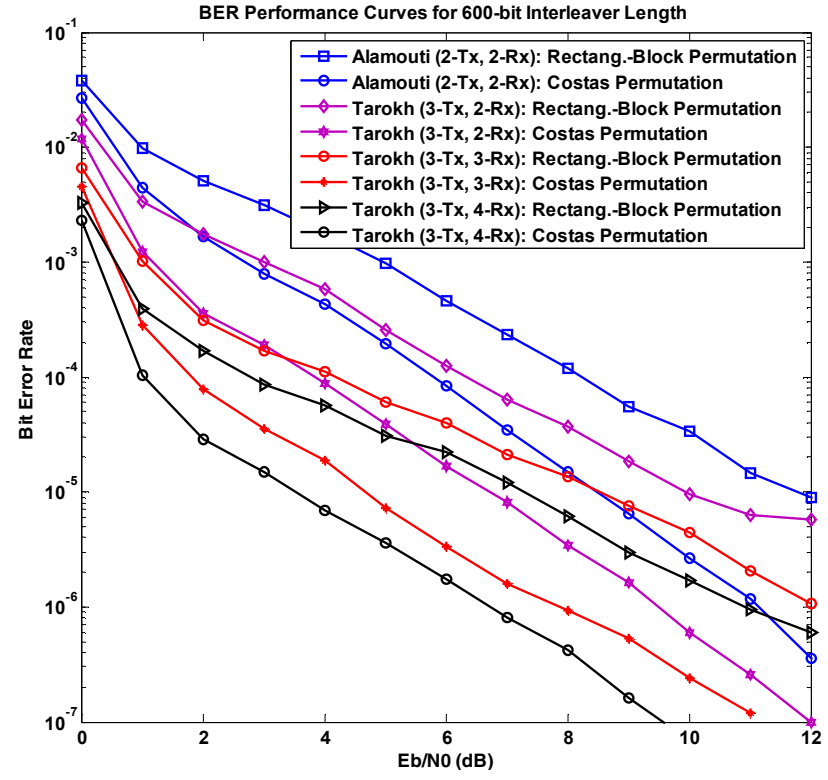

Fig. 5. Comparison of BER curves for MIMO systems with differing numbers of transmitter (Tx) and receiver (Rx) antennas, with Costas and Block permutations of $N_{I N T}=600$.

sizes $N_{I N T}=100$ and 600 . The performance curves shows that by using Costas permutation, the performance of coded MIMO systems outperform the systems utilizing optimal block interleavers (i.e. with very high and comparable depth and span which are $10 \times 10$ and $24 \times 25$ for 100-bit and 600-bit interleaver lengths respectively). The additional coding gains of $G_{d} \approx 4 \mathrm{~dB}$ for yielding $B E R \approx 10^{-6}$ in 600 -bit interleaved systems imply that the technique results in astonishing reduction of probability of bit-errors from low-to-high SNRs.

\section{CONCLUSION}

In this paper, we have shown that the BER performance of space-time turbo coded MIMO communication systems in waterfall and flare regions can be improved by applying bit-level Welch constructed Costas permutation. Our future work will focus on comparing the performance of discussed system of interest with the system employing Evolutionary Algorithm (EA) aided interleaver design that have been developed in [12], while not only compare the results in terms of BER, but also compare the computational complexities incurred by each algorithm.

\section{REFERENCES}

[1] S. M. Alamouti, "A simple transmit diversity technique for wireless communications," IEEE J. Sel. Areas Commun., vol. 16, no. 8, pp. 1451-1458, Oct. 1998.

[2] V. Tarokh, H. Jafarkhani, and A. Calderbank, "Space-time block coding for wireless communications: Performance results," IEEE J. Sel. Areas Commun., vol. 17, pp. 451-460, Mar. 1999.

[3] _ "Space-time block codes from orthogonal designs," $\overrightarrow{I E E}$ E Trans. Inf. Theory, vol. 45, pp. 1456-1467, Jul. 1999.

[4] C. Berrou, A. Glavieux, and P. Thitimajshima, "Near Shannon limit error-correcting coding and decoding: Turbo codes," IEEE ICC, Jun. 1993, pp. 1064-1070.

[5] G. Bauch, "Concatenation of space-time block codes and Turbo-TCM," IEEE ICC, Jun. 1999, vol. 2, pp. 1202-1206.

[6] T. H. Liew and L. Hanzo, "Space-time codes and concatenated channel codes for wireless communications," in Proc. of the IEEE, vol. 90, no. 2, pp. 187-219, Feb. 2002.

[7] S. Benedetto and G. Montorsi, "Serial concatenation of block and convolutional codes," Electron. Lett., vol. 32, pp. 887-888, 1996.

[8] S. Benedetto, D. Divsalar, G. Montorsi, and F.Pollara, "Serial Concatenation of Interleaved Codes: Performance Analysis, Design, and Iterative Decoding," TDA Prog. Rep., Jet Propulsion Lab., pp. 1-26, Mar. 1996.

[9] K. Enokizono and H. Ochiai, "A simple interleaver design for variable-length turbo code," $6^{\text {th }}$ Int. Symp. on Turbo Codes and Iterative Info. Processing (ISTC 2010), Sep. 2010, pp. 354-358.

[10] M. Kovaci, H. G. Balta, M. M. Nafornita, "The performances of interleavers used in turbo codes," Int. Symp. on Signals, Circuits, and Systems (ISSCS 2005), Jul. 2005, vol. 1, pp. 363-366.

[11] D.K.B. Ismail, "Towards ideal codes: looking for new turbo code schemes," PhD Dissert., Telecom Bretagne, 2011.

[12] R. G. Maunder, and L. Hanzo, "Evolutionary algorithm aided interleaver design for serially concatenated codes," IEEE Trans. Commun. vol. 59, pp. 1753-1758, Jun. 2011.

[13] F. Mehran and A. Rahimian "Physical layer performance enhancement for femtocell SISO/MISO soft real-time wireless communication systems employing serial concatenation of quadratic interleaved codes," $20^{\text {th }}$ Iranian Conf. on Elec. Eng., May 2012, pp. 1188-1193.

[14] C. Heegard and S. B. Wicker, Turbo Coding, Kluwer Academic Publishers, 1999.

[15] S. W. Golomb and H. Taylor, "Constructions and properties of Costas arrays," in Proc. of the IEEE, vol. 72, no. 9, pp. 1143-1163, Sep. 1984.

[16] K. Drakakis, F. Iorio, and S. Rickard, "The enumeration of Costas arrays of order 28," IEEE Inf. Theory Workshop (ITW 2010), Sep. 2010, pp. 1-5.

[17] K. Drakakis, "A structural constraint for Golomb Costas arrays," IEEE Trans. Inf. Theory, vol. 56, no. 11, pp. 57625764, Nov. 2010.

[18] S. W. Golomb, and G. Gong, "The status of Costas arrays," IEEE Trans. Inf. Theory, vol. 53, no. 11, pp. 4260-4265, Nov. 2007.

[19] K. Drakakis, R. Gow, and S. Rickard, "Distance vectors in Costas arrays," The $42^{\text {th }}$ Annl. Conf. on Inf. Sciences and Systems, Mar. 2008, pp. 1234-1239.

[20] A. F. Molisch, Wireless Communications, $2^{\text {nd }}$ Edition, John Wiley \& Sons Ltd., Wiley-IEEE Press, 2011.

[21] L. C. Perez, J. Seghers, and D. J. Costello, "A distance spectrum interpretation of turbo codes," IEEE Trans. Inf. Theory, vol. 42, no. 6, pp. 1698-1709, Nov. 1996. 